UDC 53.092, 532.685

S. V. Pasechnik ${ }^{1}$, D. V. Shmeliova ${ }^{1}$, A. V. Torchinskaya ${ }^{1}$, O. A. Semina ${ }^{1}$, A. A. Dyukin ${ }^{1}$, V. G. Chigrinov ${ }^{2}$

\title{
RHEOLOGICAL PROPERTIES OF LIQUID CRYSTALS IN POROUS POLYMER FILMS WITH SUBMICRON SIZES OF PORES
}

${ }^{1}$ Problem Laboratory of Molecular Acoustics, Moscow Technological University, 20 Stromynka St., Moscow, 107996, Russia. E-mail: s-p-a-s-m@mail.ru

${ }^{2}$ Hong Kong University of Science and Technology, Clear Water Bay, Kowloon, Hong Kong

The method of decay Poisenille flow was applied to study rheological properties of the nematic mixture (ZhK 440) confined to porous polymer (PET) matrix with sub micron diameters of pores. The Newtonian character of the flow was established. It made possible to determine the values of the effective shear viscosities for different temperatures. The analysis of experimental data was made taking into account the influence of weak surface anchoring effects on the orientational structure of liquid crystals.

Key words: nematic liquid crystal, porous film, anisotropic shear viscosities, Poiseuille flow.

DOI: 10.18083/LCAppl.2016.4.52

\author{
С. В. Пасечник ${ }^{1}$, Д. В. Шмелева ${ }^{1}$, А. В. Торчинская ${ }^{1}$, О. А. Семина ${ }^{1}$, А. А. Дюкин ${ }^{1}$ В. Г. Чигринов
}

\section{РЕОЛОГИЧЕСКИЕ СВОЙСТВА ЖИДКИХ КРИСТАЛЛОВ В ПОРИСТЫХ ПОЛИМЕРНЫХ ПЛЕНКАХ С СУБМИКРОННЫМИ РАЗМЕРАМИ ПОР}

${ }^{1}$ Московский технологический университет, Проблемная лаборатория молекулярной акустики, ул. Стромынка, 20, 107996 Москва, Россия. E-mail: s-p-a-s-m@mail.ru

${ }^{2}$ Гонконгский университет науки и технологии,

Залив Чистой Воды, Коулун, Гонконг. E-mail: eechigr@ust.hk

Метод затухающего потока Пуазейля был применен для исследования реологических свойств нематической смеси (ЖК 440), помещенной в пористую полимерную (ПЭТФ) матрииу с субмикронными диаметрами пор. Был установлен ньютоновский характер потока. Это позволило определить значения эффективных сдвиговых вязкостей для различных температур. Анализ экспериментальных данных выполнялся с учетом влияния эффектов слабого поверхностного сиепления на ориентационную структуру жидких кристаллов.

Ключевые слова: нематический жидкий кристалл, пористая пленка, анизотропные сдвиговые вязкости, поток Пуазейля.

(C) Pasechnik S. V., Shmeliova D. V., Torchinskaya A. V., Semina O. A., Dyukin A. A., Chigrinov V. G., 2016 


\section{Introduction}

Liquid crystals $(L C)$ are referred as partly ordered media, with translation and (or) orientational long range order intermediate between that for isotropic liquids and classical crystals. The simplest class of such media, namely nematic liquid crystals $(N L C)$ differ from isotropic liquids only by a long range orientational order described in terms of a unit vector - a director $\boldsymbol{n}$, and a scalar order parameter $S$. While the director $\boldsymbol{n}$ coincides with an overall orientation of the long molecular axes, the order parameter $S$ is characterized by the mean degree of declination of individual molecular axes from the director $\boldsymbol{n}$. The value of the parameter $S$ changes from 0 in isotropic $(I)$ phase to 1 in perfectly oriented nematic $(N)$ phase with a step-like change at the temperature $T_{c}$ of $N-I$ phase transition. It is of importance that at description of some phenomena like light scattering in an isotropic phase one has to consider the tensor order parameter Q, which incorporates both the mean direction and the degree of the orientational order [1]. The same is also true at description of some composite $L C$ systems with strong confinement [2].

Though there is no the long range order in the positions of centers of molecular masses, their translation motion described in terms of a velocity field $\mathbf{v}(\mathbf{r}, \boldsymbol{t})$ is coupled with a director field $\boldsymbol{n}(\mathbf{r}, \boldsymbol{t})$. It results in a number of new hydrodynamic effects, like flow induced orientation of $L C$, dependence of the effective shear viscosity on the flow rate (non Newtonian behavior), arising of specific hydrodynamic instabilities and a backflow under fast turning on (off) of electric fields [3]. The latter effect is of a practical importance as it can be responsible for the essential changes in operating times of liquid crystal devices.

The theoretical description of the mentioned above phenomena is based on hydrodynamic theory of Leslie-Ericksen which includes six dissipative parameters (the Leslie's coefficients $\alpha_{i}$ ), connected by one equation. Different combinations of these parameters define three principal coefficients of the shear viscosities (the Miesowicz viscosities $\eta_{\mathrm{i}}$ ) corresponding to the three different orientations of a director relatively to the directions of a flow velocity $\mathbf{v}$ and a velocity gradient $\nabla \mathbf{v}(\boldsymbol{n} \perp \mathbf{v}$, $\left.\boldsymbol{n}\left\|\nabla \mathbf{v}-\eta_{1} ; \boldsymbol{n}\right\| \mathbf{v}, \boldsymbol{n} \perp \nabla \mathbf{v}-\eta_{2} ; \boldsymbol{n} \perp \mathbf{v}, \boldsymbol{n} \perp \nabla \mathbf{v}-\eta_{3}\right)$.
The classical method of experimental determination of the Miesowicz viscosities is based on usage of strong magnetic fields, which stabilize the orientation of $N L C$, flowing through the flat capillaries [4]. In this case liquid crystals behave like anisotropic Newtonian liquid with the shear viscosity, dependant on the orientation of $L C$.

Contrary to the case of isotropic liquids, the rheological behavior of $N L C$ in Poiseuille flows through the capillaries with a circular cross section is essentially more complicated, than for flows through the flat capillaries. Nevertheless, the pioneering experiments of such type [5] provided the first experimental conformation of the Leslie-Ericksen theory. In particular, the existence of the universal dependence of the effective shear viscosity on the diameter and the instant flow rate $\mathrm{Q}=d V / d t$ ( $V$ - the pumped volume) was confirmed by experiments with a flow of $N L C$ through the capillaries of different diameters at the normal surface orientation of a liquid crystal. It is worthwhile to notice, that detailed theoretical description of the mentioned above flows is possible only by numerical solving of non linear hydrodynamic equations of $N L C$ [6].

In this paper we present the results of experimental investigations of a Poiseuille flow through the porous polymer film, which can be considered as a number of cylindrical pores of sub micron diameters. In this case different types of orientational configurations inside the pores can result in different values of the effective shear viscosity. The effects of weak surface anchoring and influence of strong confinement on dissipative parameters of $N L C$ are also may be important. In particular, the condition of strong surface anchoring, valid for glass capillaries of large enough diameters (of order $100 \mu \mathrm{m}$ ) definitely fails for the pores of sub micron diameters. It has to be taken into account at analysis of the results of rheological investigations.

The method of the decay Poiseuille flow, elaborated previously for viscosity measurements of $N L C$ stabilized by surfaces in flat capillaries [7], was successfully used in the present work.

\section{Decay flow for shear viscosity measurements}

In the simplest case a decay shear flow arises in the channel of the length $\mathrm{L}$ under the action of a hydrostatic pressure gradient, which is via the difference of levels $\Delta H$ in the open tubes of diameter $D$ connected the channel (Fig. 1). 
The flow is produced by the instant pressure difference $(\Delta P)$ slowly decreasing with time:

$$
\Delta P(t)=\rho g \Delta H(t),
$$

where $\rho-$ a density of a liquid, $g-$ a free fall acceleration. The fluid volumetric flow rate $\mathrm{Q}=d V / d t$ is the same in different cross sections of the hydrodynamic circuit and can be expressed as:

$$
\mathrm{Q}=(S / 2)(d \Delta H / d t) \text {, }
$$

where $S=\pi \mathrm{D}^{2} / 4-$ the cross section of the open tube.

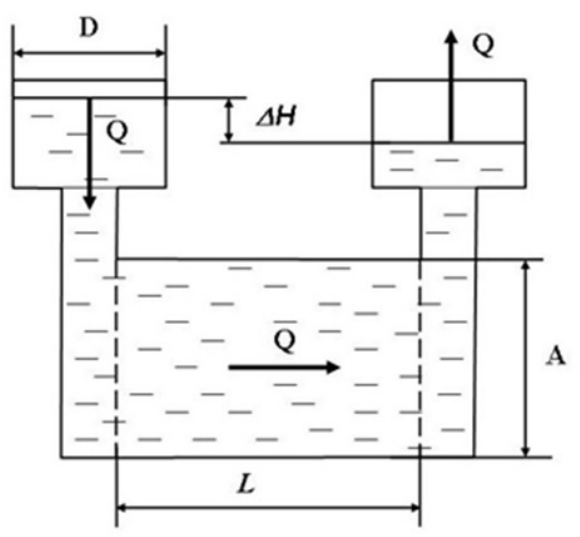

Fig. 1. The schema of a decay flow

Obviously, a certain time is required for complete cessation of the liquid motion which depends on the effective shear viscosity $\eta_{\text {eff. }}$ In the case of an isotropic Newtonian liquid $\eta_{\text {eff }}$ is equal to the shear viscosity coefficient $\eta$ and does not depend on the flow rate. It is simple to derive the next equations describing the dynamics of a decay flow of such liquid:

$$
\begin{aligned}
& \Delta H(t)=\Delta H_{\max } \cdot \exp (-t / \tau) \\
& \Delta P(t)=\Delta P(0) \cdot \exp (-t / \tau),
\end{aligned}
$$

where the characteristic decay time $\tau$ is proportional to the hydrodynamic resistance $Z$ of the channel, defined as:

$$
Z=\eta / K_{c}
$$

with the constant $K_{c}$, dependent only on the shape and the geometrical sizes of the channel. The corresponding expressions for $K_{c}$ in the cases of capillaries with the rectangular (of linear dimensions $d \times A, d<<$ ) and the circular (of a radius $r$ ) cross sections can be written as:

$$
\begin{aligned}
& K_{c}=\left(d^{3} A\right) /(12 L) \\
& K_{c}=\left(\pi r^{4}\right) /(8 L)
\end{aligned}
$$

As a result, one can get the general equation for the decay time [3]:

$$
\tau=[\eta /(2 \rho g)]\left(S / K_{c}\right),
$$

where $\rho$ - the liquid's density, $\mathrm{S}=\pi \mathrm{D}^{2} / 4$ - the cross section of the open tubes, shown in Fig. 1.

The expression (8) is also valid for a decay flow of a liquid through the sample of a porous film which is under investigation in the present work. Indeed, such sample can be considered as a number $N$ of the identical channels with a circular cross section connected in a parallel schema. In the latter case the equivalent hydrodynamic resistance $Z_{N}$ is connected with the hydrodynamic resistance $Z$ of each channel by the next trivial expression:

$$
Z_{N}=Z / N
$$

It makes possible to obtain simple generalization of the above presented expressions. In particular, the corresponding expressions for $K_{c}^{N}$ and $\tau_{N}$ can be written as:

$$
\begin{gathered}
K_{c}^{N}=\left(N \pi r^{4}\right) /(8 L) \\
\left.\tau_{N}=[\eta /(2 \rho g)]\left(S / K_{c}^{N}\right)=[\eta /(\rho g)]\left[\left(D^{2} L\right) / N r^{4}\right)\right]
\end{gathered}
$$

So, experimental measurements of a decay time $\tau_{N}$ provide determination of the shear viscosity coefficient $\eta$ in accordance with the expression:

$$
\eta=\left(\tau \rho g r^{4} N\right) /\left(D^{2} L\right)
$$

The obtained expressions (1-6) were also used previously for an investigation of rheological properties of nematic liquid crystals in a plane shear flow [7-10]. In particular, the three Miesowicz viscosities were determined via investigation of a decay flow of $N L C$ through the plane channels. In this case homogeneous bulk orientation needed for different experimental geometries was realized by a proper surface treatment of the channels [7,9] or by combined action of surfaces and strong electric fields [10]. At small values of a pressure gradient, used in experiments, the shear flow was weak enough and did not disturb the initial orientation of $N L C$. So, the Newton like behavior of liquid crystals with a constant value of the effective shear viscosity $\eta_{\text {eff }}$ took place. The similar Newtonian flow regime was realized in experiments with flows of $L C$ through the porous films described in the present paper. In the latter case, the investigation of the decay flow provides measurements of the effective values of the shear viscosities, dependant on the orientational structure of $L C$ inside pores.

\section{Experimental}

The construction of the experimental cell for a study of a decay flow is shown in Fig. 2. In many respects it is similar to the cells previously used for a 
study of linear and non-linear phenomena in liquid crystals under decay flow in flat capillaries [7-10]. The main difference is the usage of the sample of a porous PET film of thickness $23 \mu \mathrm{m}$ with a number of cylindrical pores of a constant diameter $d=2 r$, normally oriented relatively to the film. The parameters of the samples of porous films, used in experiments are presented in Table 1. The porous film separates two parts of the cell which are connected with the open vertical tubes of a diameter $D$. Preliminary PET films were treated with a solution of chromium chloride in isopropanol to provide homeotropic anchoring of a liquid crystal with the inner surfaces of pores. After filling with a liquid it was possible to impose the decay flow through the film by creating some initial difference $\Delta H_{\max }$ in the levels of the meniscuses formed in the open tubes as it shown in Fig. 1. The decay motion of the menisci was registered via a digital camera. The further analysis of images taken at different times made possible to get the dependences $\Delta H(t)$, which were compared with theory predictions. The temperature of the cell was stabilized with errors $\pm 0.1^{\circ} \mathrm{C}$.

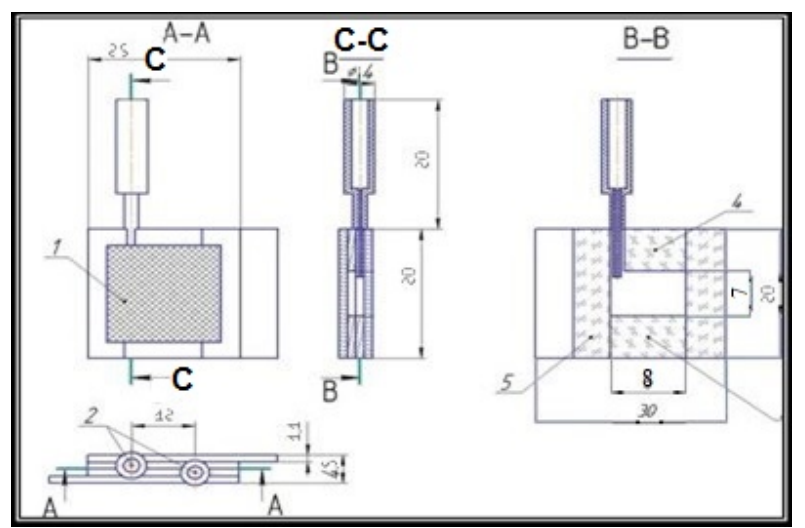

Fig. 2. The construction of the experimental cell

\section{Results and discussion}

On the first stage we studied a decay flow of ethanol which is an isotropic Newtonian liquid with well studied rheological properties. The typical time dependences $\Delta H(t)$ obtained for the films' samples with different values of the pore's diameter and the pore's density are shown in Fig. 3.

In all cases the experimental curves follow the theoretical dependence (3). It confirms the simple theory of the method and made it possible to calculate the constants $K_{c}^{N}$, needed for determination of the effective viscosity of a nematic liquid crystal.

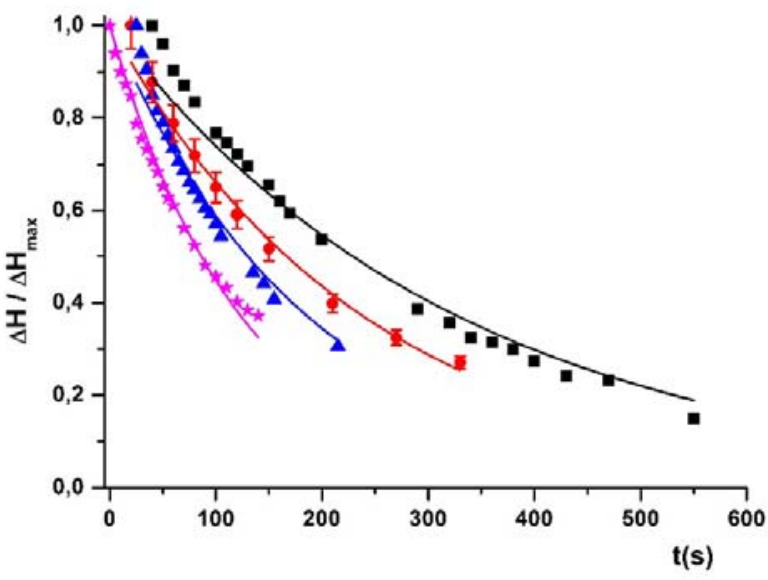

Fig. 3. The time dependence of the relative level differences for ethanol.

The diameter of open tubes $D=3.17 \mathrm{~mm}$ and diameters of pores $(d):-0.3 \mu \mathrm{m} ; \bullet-0.4 \mu \mathrm{m} ; \boldsymbol{\Delta}-0.5 \mu \mathrm{m}$; $\leftarrow-0.6 \mu \mathrm{m}$

The result of comparison between experimentally determined values $K_{c}{ }^{N}$ and those, obtained via calculation in accordance with eq. (11) is shown in Table 1.

Some disagreement can be explained by taking into account possible errors in determination of pore's diameters (up to $10 \%$ ), which may result in essential errors of the $K_{c}^{N}$ due to strong $\left(r^{4}\right)$ dependence and possible overlapping of pores. Such situation is usually met at traditional measuring shear viscosities with the help of circular capillaries.

The similar dependences of $\Delta H(t)$ in the case of a decay flow of the nematic liquid crystal for two samples with different pore's diameters at two values of temperature are shown in Fig.4.

The simple law (3) holds satisfactory for the experimental dependences. So, the Newtonian like behavior with a constant value of the effective shear viscosity $\eta_{\text {eff }}$ also takes place for NLC flow. In turn, it means that the decay flow was weak enough and did not disturb the initial orientational structure of a liquid crystal, formed inside pores by the interaction of a liquid crystal with the inner surface of the cylindrical cavities. 


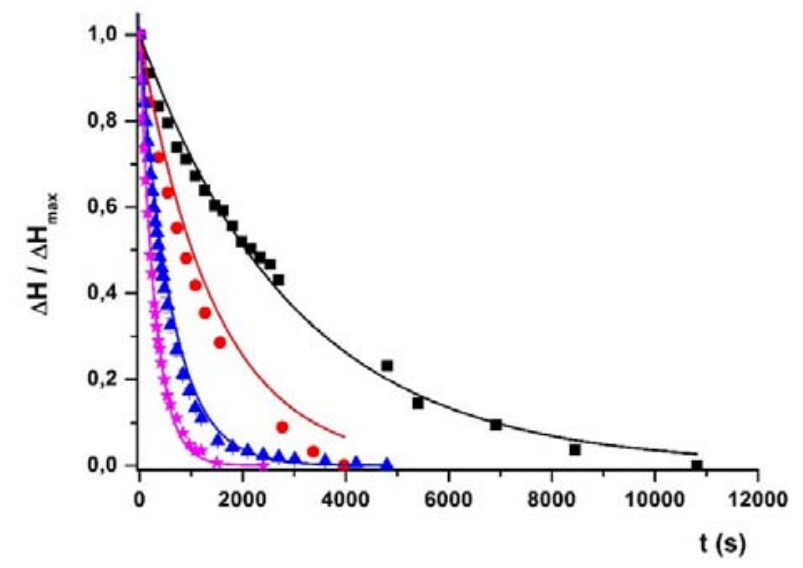

Fig. 4. The time dependence of the relative level differences for $Z h K 440$ at different temperatures.

The different values temperature $(T)$, diameters of pores $(d)$ and of open tubes $(D)$ :

$-26{ }^{\circ} \mathrm{C}, 0.6 \mu \mathrm{m}, 3.17 \mathrm{~mm} ;-42{ }^{\circ} \mathrm{C}, 0.6 \mu \mathrm{m}, 3.17 \mathrm{~mm}$;

$\Delta-26^{\circ} \mathrm{C}, 0.4 \mu \mathrm{m}, 1 \mathrm{~mm} ; \star-42{ }^{\circ} \mathrm{C}, 0.4 \mu \mathrm{m}, 1 \mathrm{~mm}$

The experimental dependences $\Delta H(t)$ and the data on $K_{c}^{N}$, obtained from experiments with an ethanol and presented in Table 1, can be used for the calculation of the values of $\eta_{\text {eff. }}$. The results of such calculations for different temperatures are presented in Fig. 5. One can compare these results with the temperature dependences of the three Miesowicz coefficients, obtained earlier for the bulk samples of $Z h K \quad 440$ [11]. It is interesting, that temperature dependence of the effective shear viscosity $\eta_{\text {eff. }}$ is close to the dependence $\eta_{3}(T)$. Such situation can take place for a planar surface orientation of a director in the plane normal to the porous axis [3]. Nevertheless, theory predicts the possibility of formation of different orientational configurations in the system under consideration $[12,13]$, which are shown in Fig. 5. The direct NMR investigations [14] of an orientational structure of a nematic inside pores of the porous polycarbonate films revealed the existence of escaped radial (ER) configuration with point defects (ERPD) and planar polar (PP) configuration dependently on the pore's diameter. Moreover, preliminary treatment of porous film by a solution of molecules providing normal surface anchoring, can induce the transition between the different configurations mentioned above.

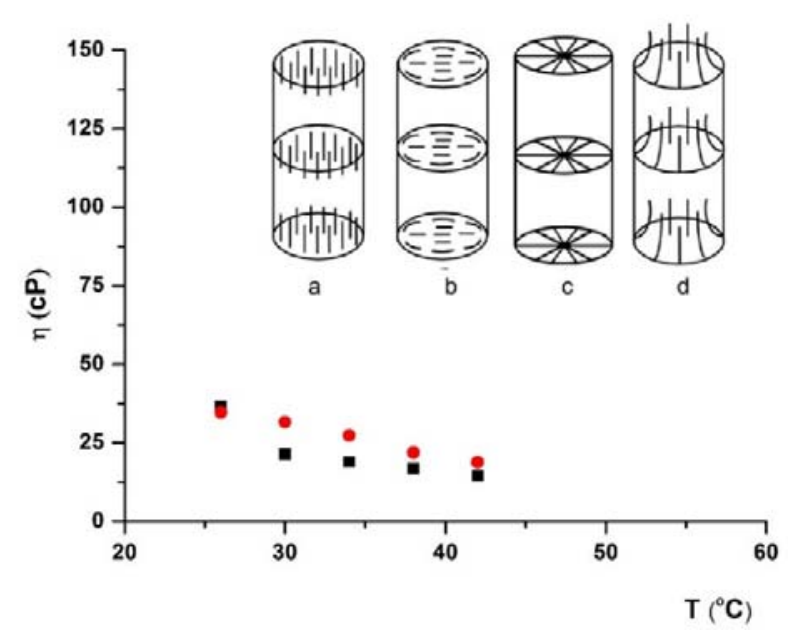

Fig. 5. The temperature dependences of results of $\eta_{\text {eff, }}$, obtained from experimental data on $\tau$ at $d$ and diameters of open tubes D: $\mathbf{\square}-0.6 \mu \mathrm{m}, 3.17 \mathrm{~mm} ;-0.4 \mu \mathrm{m}, 1 \mathrm{~mm}$. Insert - different types of director configuration: a) axial;

b) planar-polar; c) planar-radial; d) escaped radial ER

It can be used for different explanations of our experimental results. In particular, the computer simulation of ER configuration of NLC, formed inside pores [15] and marked as $(d)$ in Fig. 5, has shown that weak anchoring effects have to be taken into account for typical values of the anchoring strength and small pore's diameters. In particular, they are responsible for the declination of the surface polar angle $\theta_{\mathrm{r}}$ from the value $\theta_{\mathrm{r}}=\pi / 2$, corresponding to ideal strong anchoring. Analytic expression for $\theta_{\mathrm{r}}$ can be obtained at equal values of Frank's elastic modules $\left(K_{11}=K_{33}=K\right)$. In this case it reads as:

$$
\operatorname{Sin}^{2} \theta_{r}=\left(\sigma^{2}-1\right) / \sigma^{2},
$$

where the anchoring parameter $\sigma$ is defined as:

$$
\sigma=(r W / K)+\left(K_{24} / K\right)-1 \text {. }
$$

Table 1. The results of comparison between the values of $K_{c}^{N}$ obtained via theoretical expression (10) and those, determined from experimental data on $\tau$

\begin{tabular}{|c|c|c|c|c|}
\hline $\begin{array}{c}\text { Diameters of pores, } \\
d, \mu \mathrm{m}\end{array}$ & $\begin{array}{c}\text { decay time } \\
\tau,(\mathrm{s})\end{array}$ & $\begin{array}{c}\text { Number of the identical } \\
\text { channels, } N\end{array}$ & $K_{c}^{N}$ theor. & $K_{c}^{N}$ exp. \\
\hline 0.3 & 330.8 & $1.792 \cdot 10^{8}$ & $0.00155 \cdot 10^{-12}$ & $0.00169 \cdot 10^{-12}$ \\
\hline 0.4 & 239.8 & $0.84 \cdot 10^{8}$ & $0.00230 \cdot 10^{-12}$ & $0.00233 \cdot 10^{-12}$ \\
\hline 0.5 & 187.9 & $0.448 \cdot 10^{8}$ & $0.00299 \cdot 10^{-12}$ & $0.00297 \cdot 10^{-12}$ \\
\hline 0.6 & 124.8 & $0.224 \cdot 10^{8}$ & $0.00310 \cdot 10^{-12}$ & $0.00448 \cdot 10^{-12}$ \\
\hline
\end{tabular}


In this expression $r$ - the pore's radius, $W$ - the polar anchoring strength, $K_{24}$ - so called saddle-splay elastic module. Previous dynamic light scattering investigations of PET films, filled with a nematic (5 $C B)[16]$ have shown, that the typical values of the anchoring strength are in the range $1 \ldots .5 \mathrm{~J} / \mathrm{m}^{2}$. By taking values $W=3.510^{-5} \mathrm{~J} / \mathrm{m}^{2} . K=K_{33}=11 \mathrm{p} N[15]$, $K_{24} \approx 2 K$ (which approximately holds for nematic mixture E7 [15]) we can estimate the values of $\theta_{r}$ as $60^{\circ}$ and $51^{\circ}$ for $d=0.6 \mu \mathrm{m}$ and $d=0.4 \mu \mathrm{m}$ correspondently. The dependence of $\theta_{r}$ on $\mathrm{r}$ can result in the corresponding decreasing of the effective shear viscosity with decreasing of $r$. Such effect may be responsible for some difference between $\eta_{\text {eff. }}(T)$ dependences, obtained for the samples with different values of the pore's diameter. Indeed, in the case of ER configuration the local value of the shear viscosity depends only on the polar angle $\theta$, in an accordance with the well known expression [3]:

$$
\eta(\theta)=\eta_{2} \cos ^{2} \theta+\left(\eta_{1}+\eta_{12} \cos ^{2} \theta\right) \sin ^{2} \theta,
$$

where usually the values of the parameter $\eta_{12}$ are essentially smaller than those of $\eta_{1}$ and $\eta_{2}$. In turn, the angle $\theta$ monotonically decreases from the value $\theta=\theta_{\mathrm{r}}$ on the inner surface of a pore to the value $\theta=0$ in the centre of the pore. So, one can wait the value of the effective viscosity $\eta_{\text {eff. }}$ to be intermediate between $\eta\left(\theta_{\mathrm{r}}\right) \approx \eta_{1} \sin ^{2} \theta_{\mathrm{r}}$ and $\eta(0)=\eta_{2}$. It is possible to get, in the framework of one constant approximation, the next expression for the mean value of $\sin ^{2} \theta$ :

$$
\begin{aligned}
<\sin ^{2} \theta>=2[(1+\sigma) /(\sigma-1)][(\ln 2 \sigma /(\sigma+1))- \\
-((\sigma-1) / 2 \sigma)]
\end{aligned}
$$

The calculation, made in accordance with (15) by using the values of the parameters, presented above, results in the values $\left\langle\sin ^{2} \theta\right\rangle$ equal to 0.22 and 0.17 for $d=0.6 \mu \mathrm{m}$ and $d=0.4 \mu \mathrm{m}$ correspondently. Inserting these values into eq. (14) and using the data of independent measurements $\eta_{1}=0.12$ Pa.s, $\eta_{2}=0.02$ Pa.s, [11] one can estimate the values of the mean viscosity at room temperature as $\langle\eta\rangle=0.44$ Pa.s, and 0.39 Pa.s for $d=0.6 \mu \mathrm{m}$ and $d$ $=0.4 \mu \mathrm{m}$ correspondently. These values are close to the values of the effective shear viscosity, presented above. Nevertheless, taking into account the typical experimental errors (about $5 \ldots 10 \%$ ) in determination of $\eta_{\text {eff. }}$ it is difficult to confirm a rather weak theoretical dependence of $\langle\eta\rangle$ on $d$. Moreover, the increasing of shear viscosity with decreasing of $d$ for the porous PET films, was previously observed and assigned to the influence of strong confinement effects
[17]. These experiments were made for the films without preliminary surface treatment at more intensive shear flows. So, the further investigations are needed for detailed experimental checking of the theoretical predictions.

\section{Conclusion}

The first results of rheological behaviour of the isotropic liquid (ethanol) and the nematic liquid crystal (ZhK440) under decay Poiseuille flow through the porous PET films are obtained. It was established, that the simple exponential law predicted by a theory for Newtonian liquids holds in both cases mentioned above. In the case of NLC it corresponds to the constant value of the effective shear viscosity. The temperature dependences of the latter parameter for the samples of porous films with different pore's diameters were obtained and analysed in the frame work of theoretical results, describing the orientational structure of NLC inside pores of submicron diameters.

This work was partially supported by the Ministry of Education and Science of the Russian Federation (grant №. 3.1921.2014/K) and the RFBR (project № 16-33-00802 mol a).

\section{References}

1. De Gennes P.G., Prost J. Physics of Liquid Crystals. $2^{\text {nd }}$ ed. Oxford: Clarendon Press, 1993. 597 p.

2. Kutnjak Ž., Kralj S., Lahajnar G., Žumer S. Calorimetric study of octylcyanobiphenyl liquid crystal confined to a controlled-pore glasses. Phys. Rev. E., 2003, 68, 021705-1-021705-12.

DOI: 10.1103/PhysRevE.68.021705.

3. Pasechnik S.V., Chigrinov V.G., Shmeliova D.V. Liquid Crystals: Viscous and Elastic Properties in Theory and Applications. New York, Wiley, 2009, $436 \mathrm{p}$.

4. Kneppe H., Shneider F. Determination of the Viscosity Coefficients of the Liquid Crystal MBBA. Mol. Cryst. Liq. Cryst., 1981, 65, 23-28.

5. Fisher J., Frederickson A.G. Interfacial Effects on the Viscosity of a Nematic Mesophase. Mol. Cryst. Liq. Cryst., 1969, 8, 267-284.

6. Tseng H.C., Silver D.L., Finlayson B.A. Application of the Continuum Theory to Nematic Liquid Crystals. Phys. Fluids, 1972, 15, 1213-1222.

7. Pasechnik S.V., Chigrinov V.G., Shmeliova D.V., Tsvetkov V.A., Voronov A.N. Anisotropic shear viscosity in nematic liquid crystals: new optical measurement method. Liquid Cryst., 2004, 31 (4), $585-592$. 
8. Pasechnik S.V., Krekhov A.P., Shmeleva D.V., Nasibullaev I.Sh., Tsvetkov V.A. Orientational instability in a nematic liquid crystal in a decaying Poiseuille flow. J. Exp. Theor. Physics, 2005, 100 (4), 804-810. DOI: 10.1134/1.1926441.

9. Pasechnik S.V., Semina O.A., Shmeliova D.V., Dubtsov A.V., Chigrinov V.G., Jiatong D.V.Sun. Photo controlled surfaces in rheology of liquid crystals. Mol. Cryst. Liq. Cryst., 2015, 611 (1), 8193.

10. Pasechnik S.V., Shmeliova D.V., Semerenko D.A., Voronov A.N., Semina O.A. Modifie optical method for measurements of anisotropic shear viscosities of nematic liquid crystals. Liquid crystals and their application, 2011, 3 (37), 41-46.

11. Barnik M.I., Belyaev S.V., Grebenkin M.F., Rumyantsev V.G., Seliverstov V.A., Tsvetkov V.A., Shtykov N.M. Electrical, optical and visco-elastic properties of the liquid crystal mixture of azoxy. Crystallography, 1978, 23 (4), 805-810.

12. Bradac Ž, Kralj S, Zumer S. Molecular dynamics study of nematic structures confined to a cylindrical cavity. Phys Rev. E., 1998, 58 (6), 7447-7454.

13. Crawford G.P., Allender D.W., Doane J.W. Surface elastic and molecular-anchoring properties of nematic liquid crystals confined to cylindrical cavities. Phys. Rev. A, 1992, 45, 8693.
14. Allender D.W., Crawford G.P., Doane J.W. Determination of the liquid-crystal surface elastic constant $K_{24}$. Phys. Rev. Lett., 1991, 67 (11), 14421445. DOI: $10.1103 /$ PhysRevLett.67.1442.

15. Tkachenko V., Dyomin A.A., Tkachenko G.V., Abbate G., Sukhoivanov I.A. Electrical reorientation of liquid crystal molecules inside cylindrical pores for photonic device applications. J. Opt. A: Pure Appl. Opt., 2008, 10 (5), 055301-055306.

DOI: $10.1088 / 1464-4258 / 10 / 5 / 055301$

16. Maksimochkin G.I., Shmeliova D.V., Pasechnik S.V., Dubtsov A.V., Semina O.A., Kralj S. Orientational fluctuations and phase transitions in $8 \mathrm{CB}$ confined by cylindrical pores of the PET film. Phase Transitions, 2016, 89, (7-8), 846-855.

DOI: $10.1080 / 01411594.2016 .1199802$.

17. Tsvetkov V.A. Attempt of direct measuring of near surface shear viscosity. Mol. Cryst. Liq. Cryst., 2005, 436, 203/[1157]-216/[1170]. DOI: $10.1080 / 15421400590958034$.

Поступила в редакияию 29.11.2016 2. Received 29 November 2016 